$(Y \cdot Y \cdot$

أدب الأطفال ع ال (أغسطس الأبعاد و الآثار الاقتصادية لعمالة الأطفال

\title{
الأبعاد والآثار الاقتصادية لعمالة الأطفال في إفريقيا، ودور المنظمات الإقليمية والدولية
}

د. سالي محمد فريد

أستاذ الاقتصاد المساعد

كلية الدراسات الإفريقية العليا - جامعة القاهرة

تمثل عمالة الأطفال مشكلة خطيرة في إفريقيا؛ نتيجة أوضاع الفقر التي تعيش فيها قطاعات كبيرة من السكان، و انعدام إمكانية حصول الفقر اء على تعليم مناسب. و وعلى الرغم من وجود تشريعات تنظم عمالة الأطفال، ولكنها تفتقر إلى التنفيذ، وتعتبر البرامج الرامية إلى تخفيف حدة الفقر ، وتحسين التعليم و الخدمات البشرية ضرورية لتخفيض أشكال عمالة

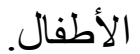

يعد العامل الاقتصادي من أهم العوامل التي تقف وراء عمالة الأطفال، ولهذا العامل

أكثر دن سبب، ولكن أهمها: الحروب، والكوارث الطبيعيـة، وتشرد الوالدين، و الهجرة غير الثرعية، وكلها تتسبب في انخفاض مستوى المعيشة، والذي يعتبر العامل الرئيس لعمالة الأطفال، والدافع لاستغلالهم، ففي بعض الأحيان بكون أجر الطفل بمثنابـة المـصدر الوحيد، أو الأساسي للاخل الذي يكفل إعالة الوالدين أو أحداهما، ويوفر الاحتياجات الأساسية التي يعجز الكبار عن نوفير ها.

تهذف هذه الورقة إلى التعرف على تطور عمالة الأطفال في إفريقيا، وتحديد الآثار الاقتصادية المترتبة عليها، ودور المنظمات الإقليمية والدولية في الحد من هذه الظاهرة، و أخيرًا تعرض الورقة عددًا من المقترحات لمواجهة عمالة الأطفال في إفريقيا.

\section{أولا ـ تطور عمالة الأطفال في إفريقيا}

شهدت القارة الإفريقية أكبر نسبة عمالة للأطفال حول العالم؛ حيث تشير

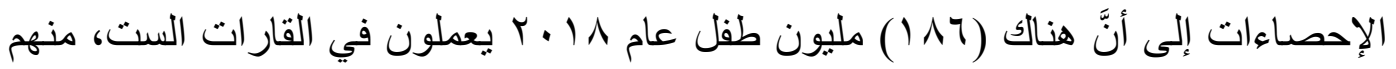
في إفريقيا وحدها (•^) مليون طفل، يعملون في أعمال خَطِرَة، ويُجنّدون في الجيوش، ويقعون فريسة للفصائل المتقاتلة، ويعتبرون ضحية لغياب الأمن وسوء الأحوال الاقتصادية و اندلاع الصر اعات العرقية و القبلية('). 


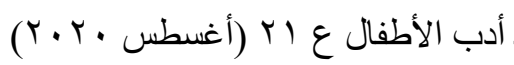
الأبعاد و الآثار الاقتصادية لعمالة الأطفال

ينخرط حوالي (·r٪) من مجموع الأطفال الأفارقة في عمالة الأطفال، وهي نسبة تزيد عن ضعفي النسبة في أي منطقة أخرى. كما بنخرط (9\%) من الأطفال الأفارقة في أعمال خطرة، وهي أعلى نسبة على مستوى العالم. ولدى إفريقيا أكبر عدد من العمال الأطفال، حيث يقدر عدد الأطفال الأفارقة المنخرطين بعمالة الأطفال بنحو ( (Y.) مليون طفل، و(0. (ب) مليون طفل في أعمال خطرة، وتقع النسبة الأكبر (0^\%) في مجالي: الزر اعة، و التعدين(؟) - (ب)

وتشير منظمة العمل الدولية إلى أن نسبة الأطفال العاملين أعلى ما تكون في إفريقيا حيث يزاول طفل من كل ثناثة أطفال في المتوسط شكلًا من أشكال النشاط الاقتصسادي، معظمه في قطاع الزر اعة. وبصفة عامة تكون مساهمة الأطفال في قوة العمل أعلى كثيرًا في المناطق الريفية منها في المناطق الحضرية، ويعمل ثناثة أرباع الأطفال العاملين في منشأة النشاط الذي تز اوله أسر هم. ونزاول نسبة ( 9\%\%) من الأطفال العاملين في المناطق الريفية أنشطة زر اعية أو أنشطة مماثلة، بينما يعمل نظر اؤهم في المناطق الحضرية بصورة رئيسة في قطاعات التجارة و الخدمات، و عدد أقل في قطاعات الصناعات التحويلية والتشييد. وعلى الرغم من أن أطفال الثوارع في المناطق الحضرية حظوا باهتمام كبير، فإن أعدادًا أكبر بكثير تعمل في الزراعة والخدمة المنزلية. وبالمنل فإن الأطفال العاملين في صناعات التصدير مثل المنسوجات و الملابس و السجاد و الأحذية قليلون نسبيَّا بالمقارنة بمن يعملون في الأنشطة الموجَّهة نحو الاستهلاك المحلي، ويرجح أن أقل من (0\%) من الأطفال العاملين يعملون في قطاعات الصناعات التحويلية أو التعدينية الموجهة للتصدير ، وأن نسبة تتر اوح بين ( (\%) و(r\%) فقط تعمل في الزر اعة الموجهة نحو التصدير (). تعد قارَّة إفريقيا إحدى القار ات الأقل استقرارًا على مستوى العالم، وذللك مع الوضع في الاعتبار كذلك منطقة شمال إفريقيا التي شهدت احتجاجات واسعة بدءًا من نهاية عام

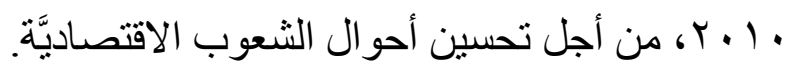

وفي هذا الإطار يقع الطفل الإفريقي بين رحى الفقر وسندان العنف المسلح، وتشير الدر اسات الاجتماعية إلى أنَّ عمالة الأطفال تؤدي إلى تشوُّهات نفسية في هؤ لاء الأطفال عند الكبر؛ حيث تزداد لايهم ميول العنف وارتكاب الجرائم، وهذا يفاقم المشكلات الاجتماعية و السياسية والأمنية الموجودة بالفعل في إفريقيا جنوب الصحر اء، والتي كثيرًا ما تَتخدم 
أدب الأطفال ع آץ (أغسطس •r.r. الأبعاد و الآثار الاقتصادية لعمالة الأطفال أولنك الأطفال أيضًا كمجندين في الصر اعات طويلة الأمد، وكذلك في المناطق قليلة السكان، بالإضافة إلى استغلالهم في أعمال شديدة الخطورة، مثل المناجم ومصانع الأسمنت (؛). كما أن زيادة الصراعات الدينية في مناطق التوترات بين المسلمين والمسيحيين،

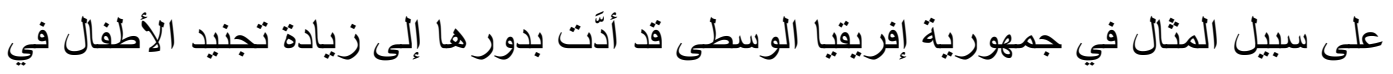

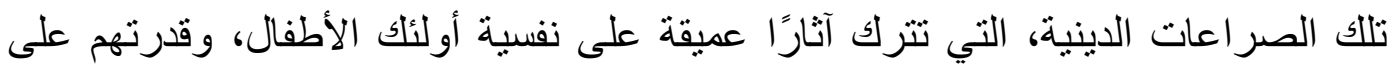

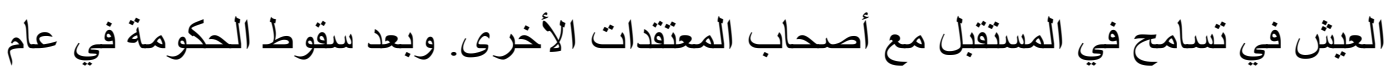

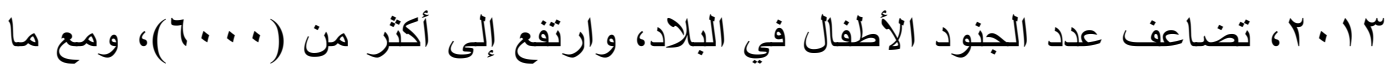

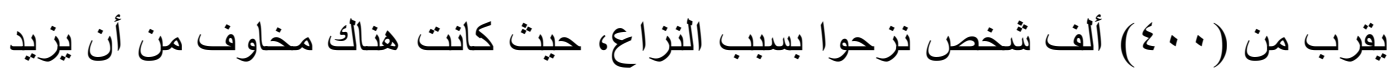

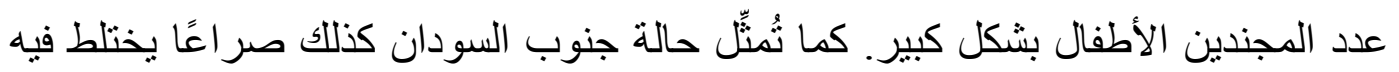
الصراع الديني بالسياسي، في ظل فنثل دولة جنوب السودان في نوفير الحد الأدنى من

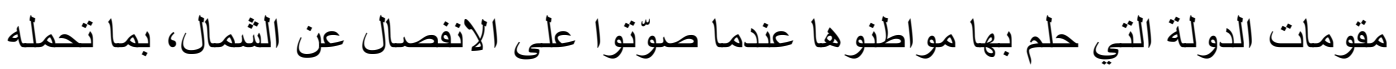
كنللك من إرث للصر اع الديني، وكذللك الصر اع على مناطق الرعي و والمياه و النفط.

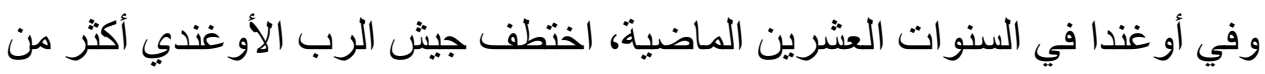
(r· ( ألف طفل، لاستخدامهم كأدو ات للقتل و التعذيب و الحرب.

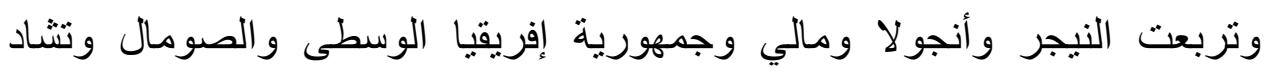

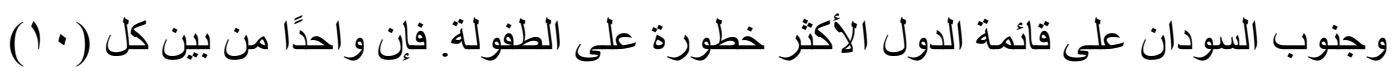

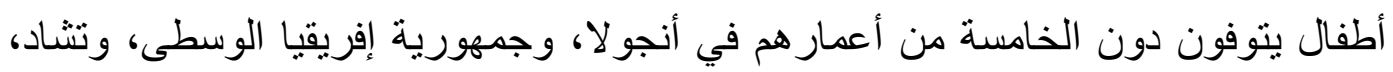

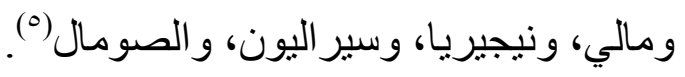
وقد أصدرت شركة "Maplecroft" للاستشارات الدولية مؤشرًا لتصنيف أسوأ الدول من حيث عمالة الأطفال، ووجد التقرير أن (7) من هذه الدول تتركز في إفريقيا، وفيما

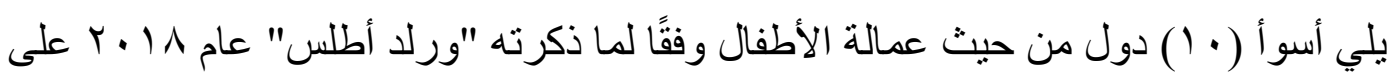
النحو التالي (T):

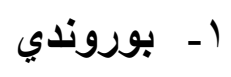

يقع أكثر من (1/\%\%) من سكان بوروندي تحت خط الفقر ، وينعكس ذللك على انتشار عمالة الأطفال إلى حد كبير، فمن بين كل (0) أطفال في الدولة هنالك واحد داخل سوق بن

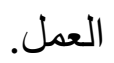




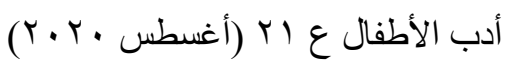
الأبعاد و الآثار الاقتصادية لعمالة الأطفال

ويعمل الأطفال في بوروندي في المنازل و المزارع و المصانع، كما يتم تجنيد الكثير

$$
\text { منهم، ويُجبرون على المشاركة في الحروب. }
$$

تنتشر عمالة الأطفال في زيمبابوي بشكل كبير ، حيث بعملون في مختلف القطاعات

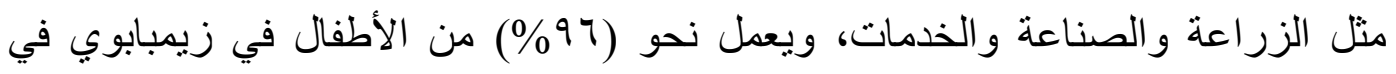
مز ارع القطن وقصب السكر و الثناي، بالإضافة إلى صيد الأسماك و التعدين و التسول.

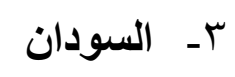

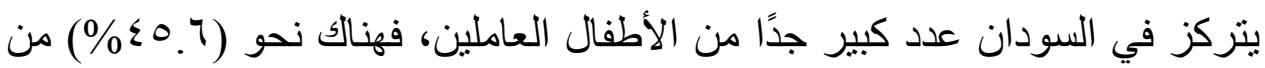

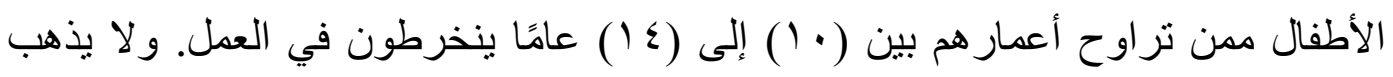

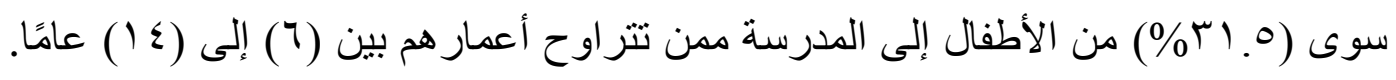

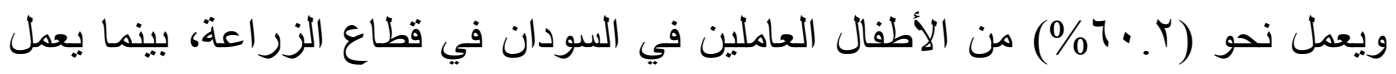

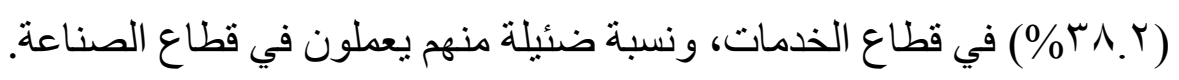

\section{ع - جمهورية الكونغو الديمقراطية}

يُجبَر الأطفال في الكونغو على العمل في مناجم الذهب و الولفراميت و الكولتان، إلى

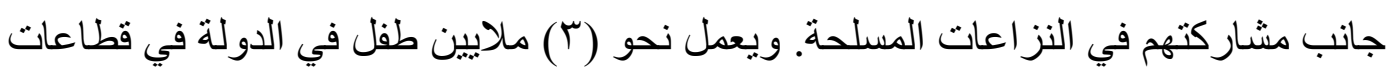
عديدة من بينها الزر اعة و الصناعة و الخدمات، ويتم تجنيد الأطفال قسرًا في الجيش بالدولة. هـ الصومال

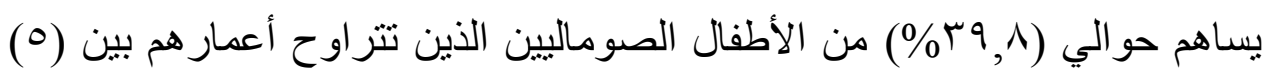

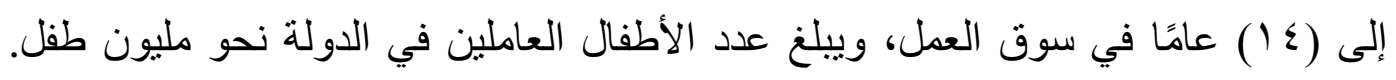

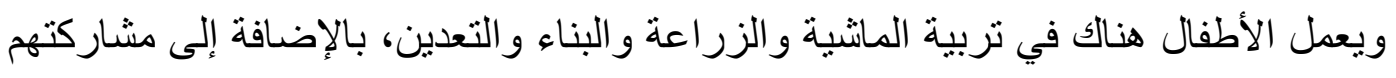
في النزاعات المسلحة والأنشطة غير القانونية، كما يتعرضون للنية للاستغلال الجنسي والإتجار بهم.

\section{- - إريتريا}

تصدرت إريتريا القائمة، حيث تطبق الحكومة بر امج يُطلب بموجبها من الأطفال في

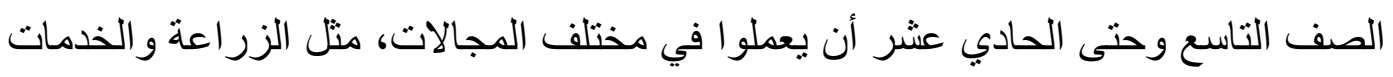

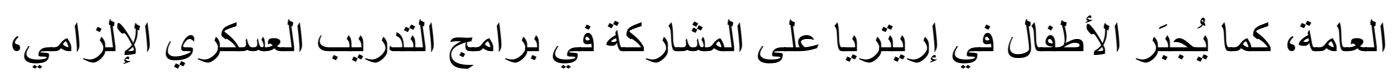




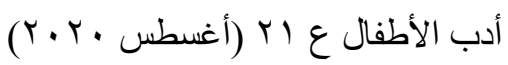
الأبعاد والآثار الاقتصادية لعمالة الأطفال

ورغم وجود قو انين لمنع عمالة الأطفال إلا أنها لا تُطبّنق، فالعمل القسري و استغلال الأطفال جنسيًا منتشر على نحو كبير في الدولة.

\section{ثانيًا ـ الآثار الاقتصادية لعمالة الأطفال في إفريقيا}

يعزز الفقر ومشاركة الأطفال في النشاط الاقتصادي كل منهما الآخر. وينسبب الفقر

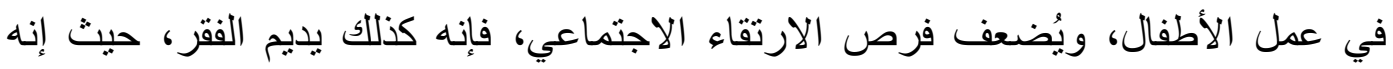

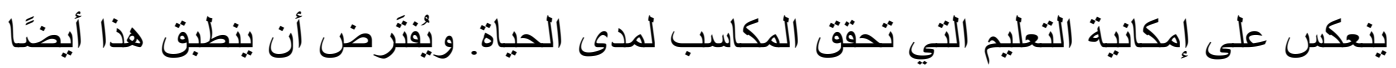

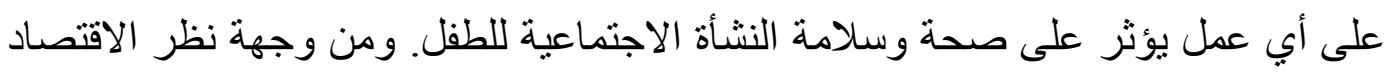

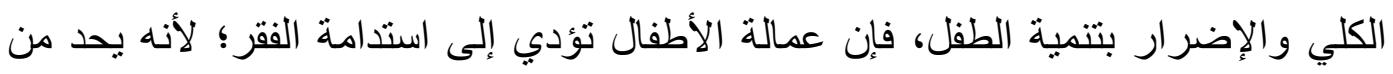

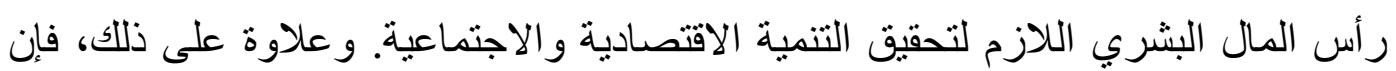
مشاركة الأطفال في النشاط الاقتصادي يزيد الفقر؛ لأنه يزيد البطالة ونقص عمالة البالغين.

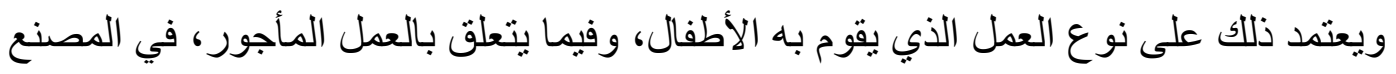

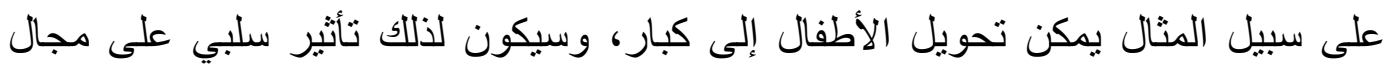
العمل، والأجور، وظروف العمل الأخرى للبالغين. كما أن عمل الأطفال له آثار تمييزية

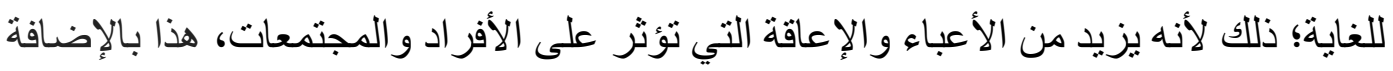

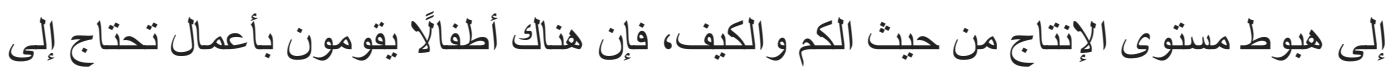
الاقة، خاصة في مجالي التنيبيد و البناء. وكذلك تضخم حجم قطاع الخدمات غير الإنتاجية('). وتثير الأدبيات الاقتصادية إلى أن معدل انتشار عمالة الأطفال و عدد الأطفال كنسبة

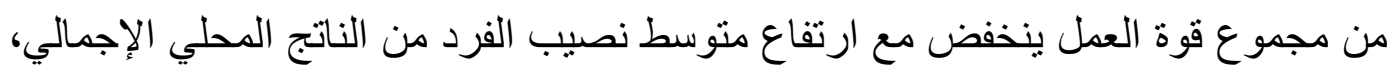

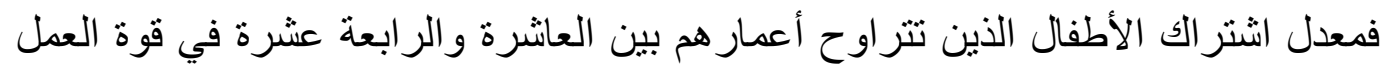

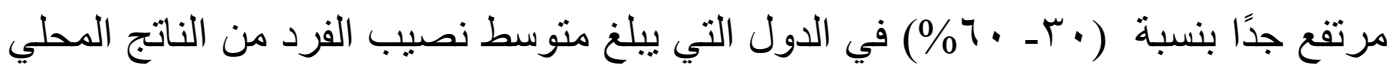

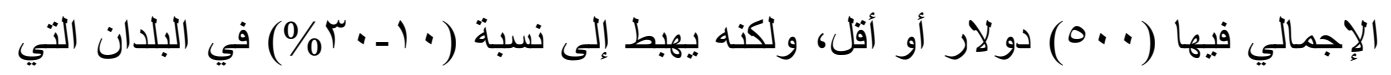

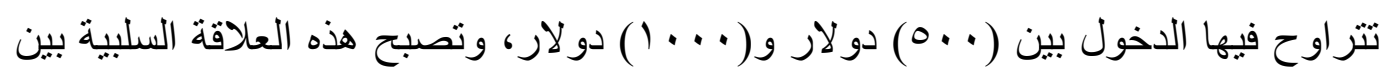

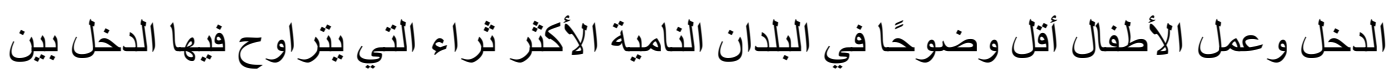

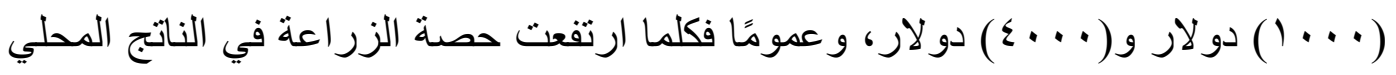

الإجمالي، زاد معدل انتشار عمالة الأطفال(^). 


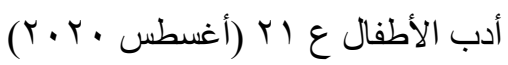
الأبعاد و الآثار الاقتصادية لعمالة الأطفال كما يتضح من الأدبيات الاقتصادية انخفاض معدل انتشار عمالة الأطفال مع ارتفاع معدل القيد في المدارس، وقد تكون هذه العلاقة غير و اضحة إلى حد ما؛ لأن بعض الأطفال

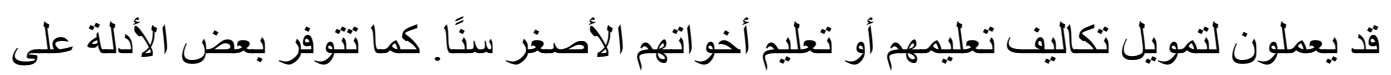

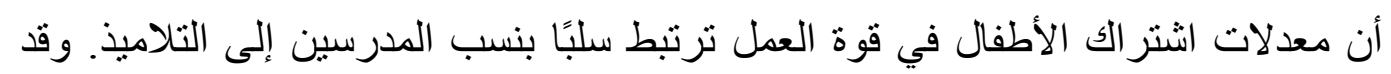

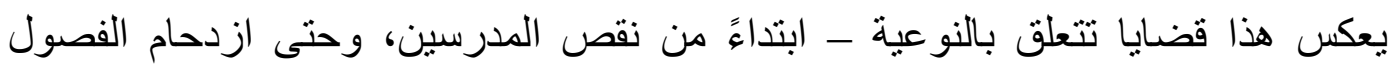
الدراسية - وينتج عنها انخفاض التوقعات المتعلقة بالقيمة المضافة من التعليم، و انخفاض

العو ائد الخاصة المتوقعة من تعليم الأطفال(9). تعد آثار النمو، وتخفيض أعداد الفقراء، وتوسيع نطاق التعليه، والحد من النمو لأن

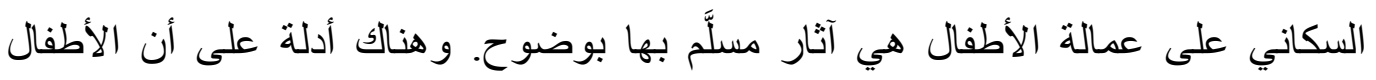

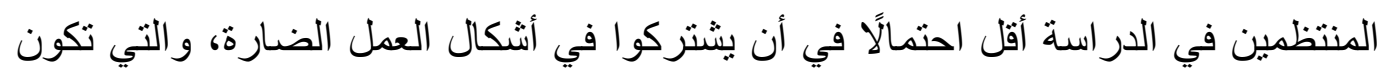
معظمها غير متسقة مع الانتظام العادي في الدراسة. وتثير الإحصاءات إلى أن (9) ملايين من الأطفال الإناث، و(7) ملايين من الذكور متخلفون عن المدارس في الدول الإفريقية

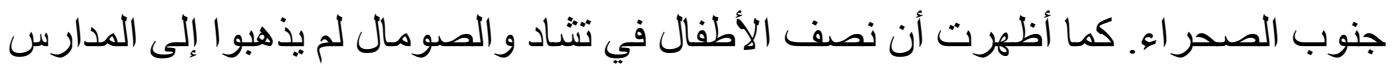
أبدًا، فيما تشكل نسبة الأطفال المحرومين من حق التعليم في النيجر (00\%)، وجيبوني

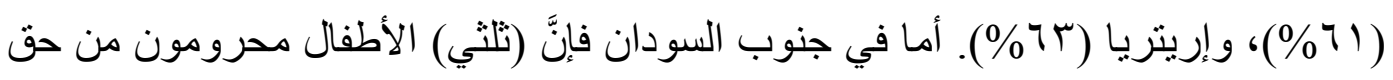

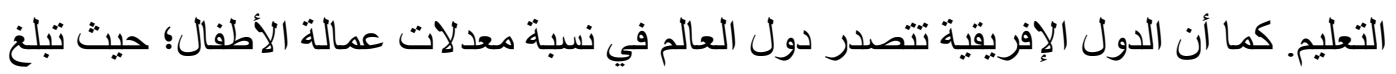

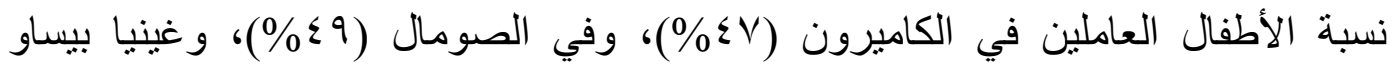

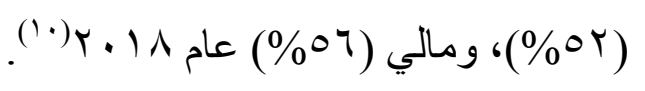

وتُثير هذه الإحصاءات القلق؛ حيث إنها تقترب أو تزيد عن النصف في الكثير من

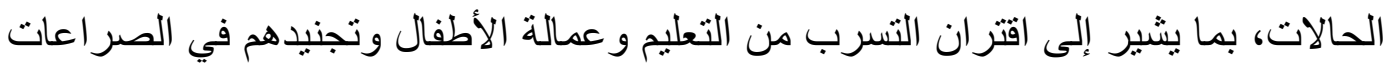

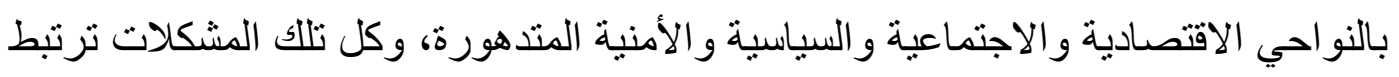

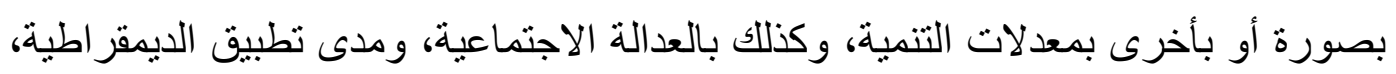

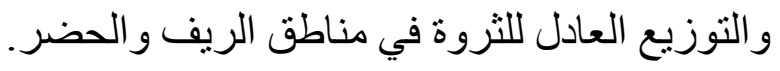

ثالثًا ـ دور المنظمات الإقليمية والدولية في الحد من عمالة الأطفال:

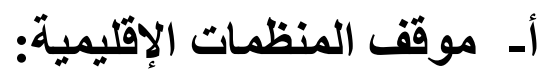

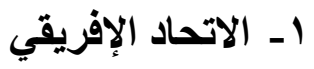




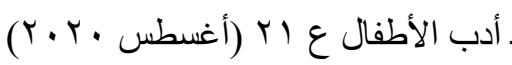
الأبعاد و الآثار الاقتصادية لعمالة الأطفال -

اعتمدت مختلف الدول الإفريقية خطط عمل وطنية للقضاء على عمالة الأطفال. ومع ذللك، و على الرغم من هذه الجهود والتراجع العالمي في عمالة الأطفال، فمن المؤسف أن معظم البلدان التي تعاني من أسو أ ممارسات عمالة الأطفال توجد في إفريقيا، والتي تعد من بين أكثر المناطق تأثرًا بحالات هشاشة وأزمات الدولة، الأمر الذي أدى بدوره إلى زيادة خطر عمالة الأطفال؛ لذا انطلقت المشاورة الفنية حول مشروع خطة عمل الاتحاد الإفريقي العشرية للقضاء على عمالة الأطفال، و العمل القسري، والإتجار بالبشر، و العبودية، بتاريخ با فبر اير 9 ا ـ ب، كجزء من جهود الاتحاد الإفريقي لتحقيق أهداف وتطلعات أجندة التنمية الإفريقية با • r بشأن القضاء على كافة أشكال عمالة الأطفال في القارة، بما يتماثى مع

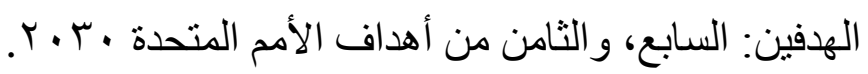

\section{r ـ المجلس العربي للطقولة والتنمية}

قام المجلس بتفيذ وتقييم بر امج تدخل لمواجهة مشكلة عمل الأطفال، بالتعاون مع برنامج "إيبك" التابع لمنظمة العمل الدولية، وبرنامج الخليج العربي للتنمية "أجفند". وتم إعداد الاستراتيجية العربية للحد من عمل الأطفال، بالتعاون مع منظمة العمل العربية وجامعة الدول العربية، بالإضافة إلى المشروع العربي لحماية أطفال الشوارع، ومشروع سياسات حماية الطفل العربي، ومشروع إعلام داعم لنشر ثقافة حقوق الطفل، ومناهضة العنف ضد الأطفال(').

\section{بـ-موقف المنظمات الدولية}

يقوم البنك الدولي بتفويض المنظمات الدولية المتخصصة، ويتيح لها ذلك اتباع نهج أكثر تركيزًا على قضايا عمالة الأطفال بشكل عام. فعلى سبيل المثال تختص منظمة اليونسكو بالتربية والتعليم، وتتحمل منظمة اليونيسيف مسؤولية عالمية عن تحسين وضع الأطفال؛ وتختص منظمة العمل الدولية بالتوظيف وقضايا العمالة الأوسع نطاقًا على أساس ثناثي، بينما هدف البنك الدولي هو مساعدة الدول المقترضة، الأعضاء فيه في جهود الإعمار والتتمية الاقتصادية والاجتماعية. وبالإضافة إلى ذلك يوجد اتفاق في الآر اء على أن الشر اكة على المستوى الدولي والقطري تمثل شرطًا مسبقًا لمكافحة عمالة الأطفال، وكثيرًا ما تسعى المنظمات الأخرى إلى التعاون مع البنك الدولي (rا'. 


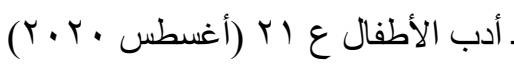
الأبعاد و الآثار الاقتصادية لعمالة الأطفال

\section{1 - 2 - منظمة اليونيسيف}

تدعم اليونيسيف خارطة الطريق للقضاء على أسوا أشكال عمالة الأطفال، و التي تدعو إلى استجابة متكاملة لعمالة الأطفال. كما تدعم اليونيسيف المجتمعات المحلية في تغيير قبول عمالة الأطفال ثقافيًا، وفي نفس الوقت تدعم استراتيجيات وبر امج توفير دخلٍ بديلٍ للعائلات، و الحصول على خدمات دور الحضانة والتعليم الجيد و الخدمات الوقائية. وتعمل اليونيسيف أيضًا مع أصحاب العمل والقطاع الخاص على تقييم سلاسل

إمداداتهم وممارساتهم التجارية، ودراسة تأثير ها على الأطفال. وقد أعدت منظمة (ئت اليونيسيف) قائمة مراجعة "للخيارات الاستراتيجية التي يمكن أن تحقق أفضل مصالح للطفل". وبالنسبة لعمالة الأطفال وأثنكال الاستخلال الاقتصادي الأخرى للأطفال، تتضمن القائمة: زيادة فرص التعليم، إما عن طريق أخذ إجازة للانتظام في الدراسة، و إما عن طريق توفير التعليم في مكان العمل؛ وتوفير خدمات إعالة لأولياء الأمور، خاصة الأمهات؛ وتشجيع التشدد في تطبيق القو انين ضد المتاجرين بالأطفال، ومن يبرمون "عقود إذعان" في مجال عمالة الأطفال؛ وتوفير خدمات للأطفال الذين يعملون في الشوارع، ورفع سن الزواج، وتغيير القيم الثقافية والأعراف الاجتماعية التي تتساهل إز اء الاستغلال الاقتصادي

لكأطفال(') (1T).

\section{ب - ب - منظمة العمل الدولية}

قامت المنظمة باتخاذ إجراءات على المستوى القطري، وتحسين التعاون الدولي. فعلى المستوى القطري تدعو المنظمة إلى استراتيجية وطنية وإجراءات محددة لمكافحة عمالة الأطفال؛ لتحسين قو اعد البيانات الخاصة بعمالة الأطفال، لتوفير معرفة، وتشخيص أفضل للوضع، وزيادة الوعي بمشكلة عمالة الأطفال، و إقامة تحالف اجتماعي عريض ضد عمالة الأطفال، وخلق قدرات مؤسسية للتعامل مع مشكلة عمالة الأطفال. وتدعو منظمة العمل الدولية البلدان الموقعة على اتفاقيات المنظمة ذات الصلة أو اتفاقية الأمم المتحدة المعنية بحقوق الطفل إلى اعتماد برامج عمل محددة زمنيًا للقضاء على عمالة الأطفال. وتشمل الإجراءات المحددة التي اقترحتها منظمة العمل الدولية تحسين التشريعات الخاصة بعمالة الأطفال، و إجر اءات تطبيقها، وتحسين وتوسيع نطاق التعليم المتاح للفقر اء، واستخدام حو افز اقتصادية "إيجابية" لتخفيض المعروض من عمالة الأطفال، كما أن منظمة العمل الدولية تحذر من استخدام الحوافز "السلبية" مثل العقوبات التجارية، ومن آثار ها غير 


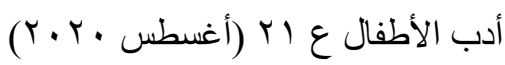
الأبعاد والآثار الاقتصادية لعمالة الأطفال المقصودة على الأطفال العاملين، و أنه بدلًا من فرض عقوبات تجارية أو التهديد بفرضها

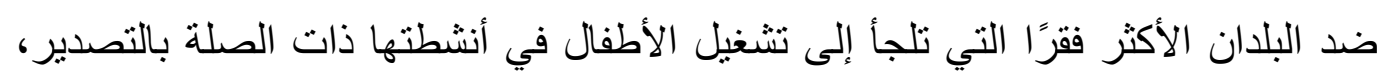
يجب على البلدان المتقدمة أن تساعد هذه البلدان الأكثر فقرًا في حل مشاكلها المتعلقة بعمالة الأطفال() (1).

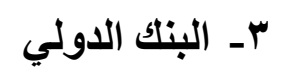

يبذل البنك الدولي مزيد الجهود في مجالات التعليم و الصحة و السكان، و التي تساعد

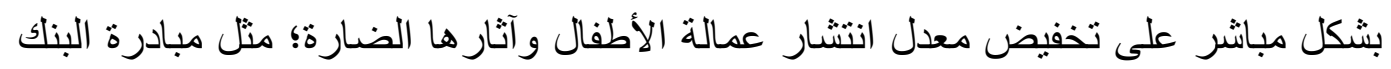
الخاصة بالأطفال، التي ترصد الأنشطة المتعلقة بالأطفال في مجالي: الصحة، والتئل التعليم، وينت

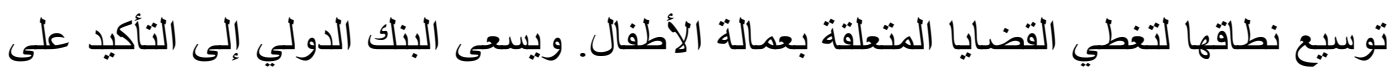

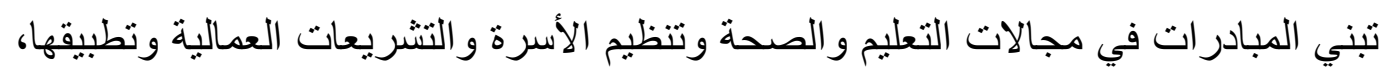

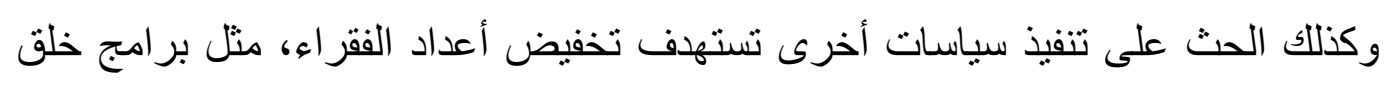
فرص العمل، وزيادة الدخل(10).

\section{رابعًا ـ وسائل تخفيض عمالة الأطفال في إفريقيا}

هناك عدة سبل مقترحة لمكافحة عمالة الأطفال، وتتمثل في تخفيض أعداد الفقر اء،

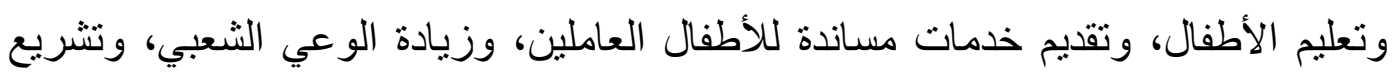

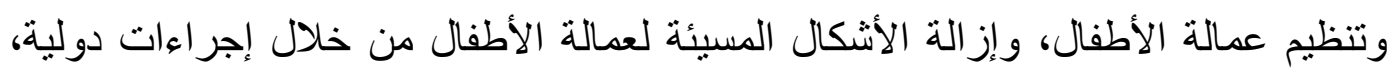

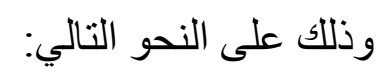

\section{1- تخفيض أعداد الفقراء}

يُعَدُّ انتشار الفقر أحد الأسباب الرئيسة في عمالة الأطفال، ففي الأسر الفقبرة، قد

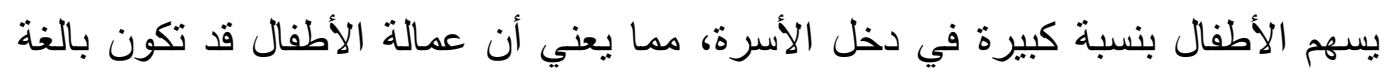

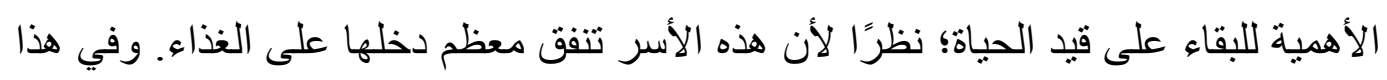

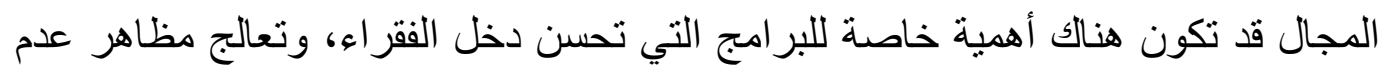
اكتمال أسواق ر أس المال، وتوفر شبكات أمان (1').

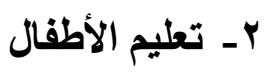

إن الإجراءات التي تستهدف زيادة الاشتر الك في التعليم الابتدائي تثجع تنمية رأس

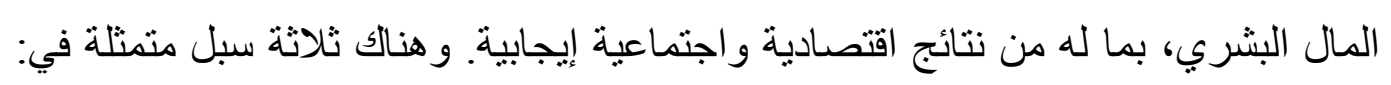

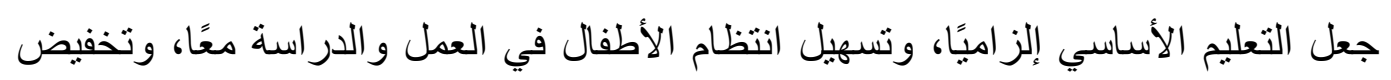




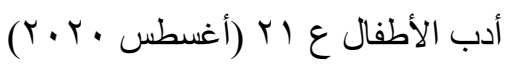
الأبعاد و الآثار الاقتصادية لعمالة الأطفال التكاليف التي تتحملها الأسر مقابل انتظام الأطفال في الدراسة. ويعتبر التعليم الإلزامي

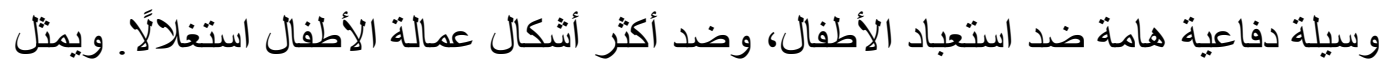
تخفيض تكاليف التعليم خيارًا آخر؛ لأن ذلك يعطي حافزًا للأسر لتحويل المعروض من

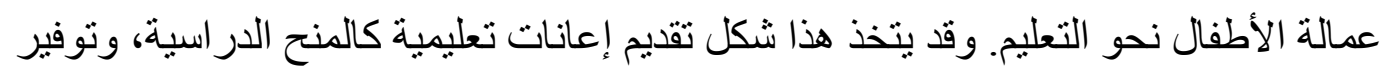
المستلزمات التعليمية(') (1).

\section{r- ت تقديم خدمات مساندة للأطفال العاملين}

تسعى معظم هذه المبادرات إلى مساعدة الأطفال الذين يعملون في القطاع الحضري

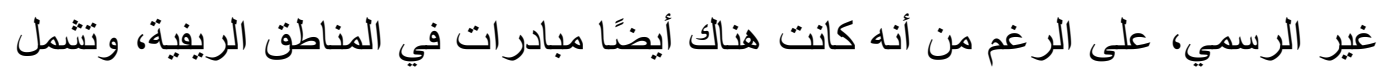

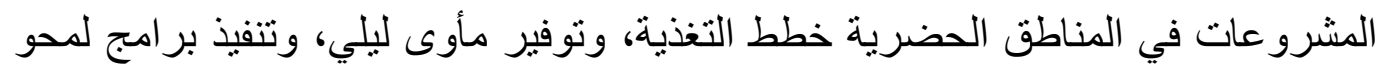
الأمية، وتنفيذ برامج أخرى، كما تسعى بعض المشروعات إلى مساعدة أسر الأطفال

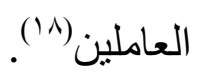

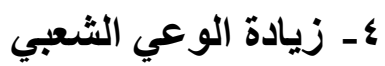

يغطي الوعي الثعبي نطاقًا عريضًا من الأنشطة التي تشمل: تحسين معرفة الأطفال

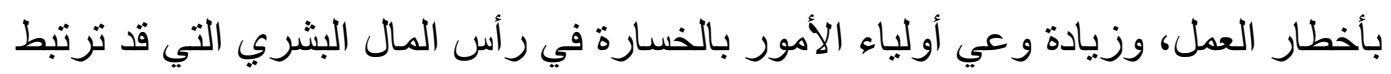

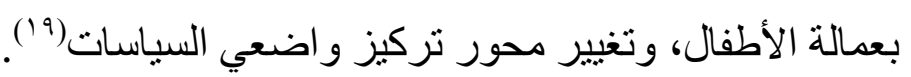
هـ التشريع والتنظيم

لاى معظم الدول تثريعات ولو ائح منظمّمة لعمالة الأطفال، ولكن كثيرًا ما يكون تطبيقها محدودًا. وهناك إدراك متز ايد بأن التشدد في التطبيق العام، في ظروف كثير من الدول، قد يلحق الضرر بمن يستهدف التطبيق حمايتهم، عن طريق تخفيض دخول الأسر

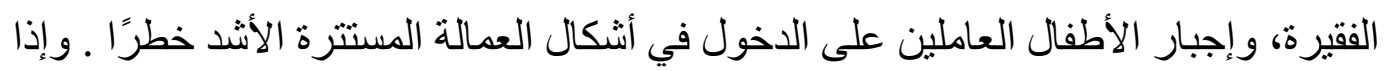
كانت رواتب المفتشين منخفضة، فإن احتمال الرشوة وتطبيق التشريعات على أساس تقديري

قد يصبحان خطرًا حقيقيًا (r).

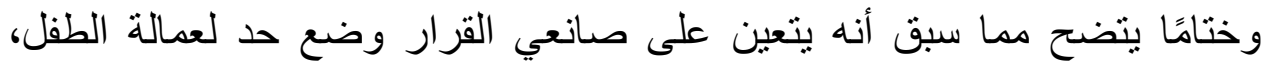

بالإضافة إلى القضاء على الفقر، حيث تعد القطاعات الأشد فقرًا و المحرومة هي المصدي لهدر

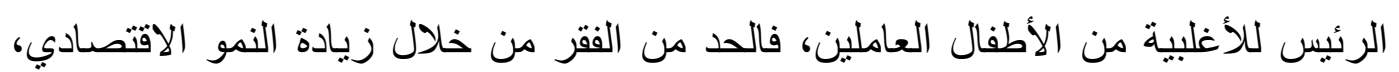

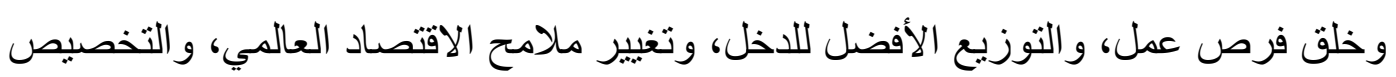




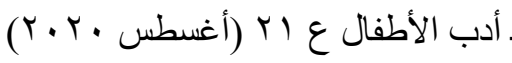
الأبعاد و الآثار الاقتصادية لعمالة الأطفال

الأفضل للموازنات الحكومية، و التوجيه الأفضل للممتلكات الخاصة ـ من شانها أن تقلل من معدلات الفقر؛ وبالتالي تقليص عمالة الطفل للحد الأدنى.

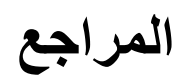

’. ILO :"Facts on Child Labor - r• ^^" (PDF). Geneva.

r. Ibid.

r. ILO "Child labor in Africa" (PDF). r • 10.

ะ. Canagarajah, R.S., and H. Columbe. $r \cdot 10$. "Child Labor in Africa"

•. Karen Wells, Findings on the Worst Forms of Child Labor r.17.

ๆ. Maplecroft | Global Risk Data, Country Analysis \& Consulting, WWW.maplecroft.com

v. Grootaert, Christiaan, and Ravi Kanbur. "Child Labor: A Review." World Bank Policy Research Working Paper I $\leqslant$. Washington, D.C.

^. Ibid.

१. Siddiqi, Faraaz, and Harry Patrinos. Causes and

a. Interventions." Human Resources Development Working Paper No. 07. World

b. Bank, Washington, D.C.

1. Ibid.

WWw.arabccd.org المجس العربي للطفولة و التنمية

IT. World Bank: Human Development r, Washington, D.C.

Ir. UNICEF : The State of The World's Children - Y. II" (PDF). $r \cdot 1 r$. 


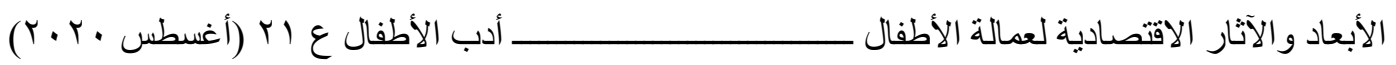

I . ILO: "National programme on the elimination of child labour" (PDF). r. 10.

10. World Bank: Children's Rights." International Journal of Children's Rights. vol. $\leqslant$.

17. Canagarajah, R.S., and H. Columbe, op.cit.

Iv. Karen Wells, op.cit.

1^. Siddiqi, Faraaz, and Harry Patrinos, op.cit.

19. Ibid.

$r \cdot \quad$ Grootaert, Christiaan, and Ravi Kanbur, op.cit. 